\section{Rehabilitation of the upper extremity and basic activities of daily living in the first month after ischemic stroke: an international cohort comparison study}

\author{
Cesar Minelli, ${ }^{1,2}$ Francisco A.A. Gondim, ${ }^{2,3}$ \\ Amilton Antunes Barreira,' \\ Alexander W. Dromerick, ${ }^{2}$ \\ 'Departamento de Neurologia, Psicologia \\ e Psiquiatria, Universidade de São Paulo, \\ Ribeirão Preto, São Paulo, Brazil; \\ 2Department of Neurology, Washington \\ University School of Medicine, St. Louis, \\ Missouri, USA; \\ ${ }^{3}$ Departamento de Fisiologia e \\ Farmacologia \& Neurology Division, \\ Hospital Universitário Walter Cantídio, \\ Universidade Federal do Ceará, \\ Fortaleza, Ceará, Brazil
}

\section{Abstract}

Inpatient rehabilitation has been traditionally employed in developed countries, while in developing countries, outpatient rehabilitation is the rule. The purpose of this study was to compare the patterns of recovery of upper extremity (UE) function, global impairment and independence in activities of daily living (ADL) during the first month after ischemic stroke in inpatient (United States) and outpatient (Brazil) rehabilitation settings.

This is a prospective cohort comparison study. Twenty patients from each country were selected using identical inclusion criteria.

The study measures employed were the UE portion of the Fugl-Meyer scale, the Action Research Arm test, the National Institutes of Health Stroke Scale and Barthel Index. Changes from baseline to the end of treatment, efficiency and effectiveness of each treatment were compared.

Both populations exhibited significant improvement between the first and second evaluations in the four outcome scales $(p<0.0001)$. There were no differences between the two rehabilitation settings on any of the four dependent measures $(p>0.05)$.

Substantially different treatment approaches after ischemic stroke led to similar results in UE function, global impairment and ADL. Further studies in larger populations should be performed in order to confirm the present results.

\section{Introduction}

Stroke is a leading cause of neurological disability in the western world ${ }^{1}$ and affects health care systems globally. There are great variations between countries in how stroke rehabilitation care is delivered. Choices are driven by public health, cultural, political, and economic circumstances. ${ }^{2-4}$

In developed countries, an inpatient rehabilitation stay is typical, and occurs after an acute hospitalization that can vary from 3-48 days after stroke. ${ }^{5}$ These inpatient treatments can include an organized multidisciplinary setting, incorporating a team of physicians, nurses and therapists or may occur in general wards without a coordinated, multidisciplinary team care. ${ }^{6}$ In contrast, resources for rehabilitation are more limited in developing countries, where most patients may undergo restricted outpatient rehabilitation, or receive no organized rehabilitation.

Meta-analyses have concluded that organized inpatient stroke care is more likely to reduce death, the odds of institutionalization and improves independence when compared to the care provided on general wards. ${ }^{7}$ Metaanalyses comparing inpatient and daily outpatient rehabilitation after the acute stroke phase have shown that the functional independence is similar. ${ }^{8,9}$ Some studies have reported that outpatient strategies may be more advantageous in terms of increased personal satisfaction, ${ }^{10}$ a shorter hospital stay and lower costs. ${ }^{11-13}$

However, to our knowledge, no study has compared the recovery of stroke patients under organized inpatient with outpatient rehabilitation (twice a week). Therefore, we compared the patterns of motor recovery in the upper extremity (UE), global impairment and independence in basic activities of daily living (ADL) throughout the first month after ischemic stroke in patients who underwent a typical U.S. organized inpatient rehabilitation stay with similar patients who received the twice a week outpatient rehabilitation typically delivered in parts of Brazil. We hypothesized that patients who met the criteria for organized inpatient rehabilitation in the U.S. would show better patterns of recovery than the outpatient rehabilitation setting in Brazil.

\section{Design and Methods}

\section{Study design}

This was a prospective cohort comparison study which included a group of subjects undergoing inpatient ischemic stroke rehabilitation in the United States and a group of sub-
Correspondence: César Minelli,

Hospital Carlos Fernando Malzoni, 556-Centro, Matão, SP, Brazil.

E-mail: cminelli@netsite.com.br

Key words: rehabilitation, arm, activities of daily living, outcome assessment, international randomized controlled trials.

Acknowledgments: this work was supported in part by a grant from the United States National Institutes of Health, 1 R01 NS 41261, the James S. McDonnell Foundation and by the Foundation for the Coordination of Higher Education and Graduate Training, (CAPES), Brazil.

Disclosures: there is no conflict of interest with pharmaceutical companies, biomedical device manufacturers, or other corporations whose products or services are related to the subject matter of the article. Also, there is no conflict of interest with honoraria, consulting fees, grants or funds from corporations.

Received for publication: 27 March 2009.

Accepted for publication: 4 May 2009.

This work is licensed under a Creative Commons Attribution 3.0 License (by-nc 3.0)

(C) Copyright C. Minelli et al., 2009

Licensee PAGEPress, Italy

Neurology International 2009; 1:e4

doi:10.4081/ni.2009.e4

jects with similar clinical characteristics undergoing outpatient therapies in Brazil. Both groups were selected using identical inclusion criteria, and both received the local standard of care for post-stroke treatment. The Institutional Review Boards of both universities reviewed and approved this study. Only individuals able to provide informed consent were included. Data from the inpatient rehabilitation group was collected in the U.S. between April and October 2002. Data from the outpatient group was collected in Brazil between November 2002 and May 2003.

\section{Patients}

Patients were selected according to the following inclusion criteria: 1) ischemic stroke confirmed by neuroimaging; 2) score of at least 1 in the UE item of the National Institutes of Health Stroke Scale (NIHSS); 3) maximum of 14 days between stroke onset and the first evaluation; 4) no history of stroke or no residual deficits from prior stroke; 5) ability to follow three step commands with the less affected UE; 5) ability to provide informed consent. Individuals were excluded if they had: 1) active serious mental disorder or delirium; 2) presence of important visual-spatial deficit as measured by a score of greater than 1 on the NIHSS neglect item 3 ) less than a two-week inpatient 
rehabilitation stay (U.S. group) 4) stroke between evaluations, and 5) UE amputation.

The cohort receiving organized inpatient rehabilitation care was recruited at the Healthsouth Rehabilitation Institute of St Louis, a for-profit academic rehabilitation hospital in St. Louis, Missouri, USA, a city of 350,000 people embedded within a metropolitan area of 2.6 million people. Individuals are typically admitted for inpatient rehabilitation within a few days of stroke onset, and undergo a coordinated, interdisciplinary rehabilitation program provided by a specialized stroke rehabilitation service. Most undergo outpatient therapy treatment after discharge from inpatient rehabilitation. More than $90 \%$ of stroke patients are referred from Barnes-Jewish Hospital, a non-profit tertiary care inner city teaching hospital. Fifty-eight consecutively admitted ischemic stroke patients were assessed by the investigators between April and October 2002. Twenty subjects met the eligibility criteria and were enrolled.

The cohort receiving outpatient rehabilitation twice a week was recruited at the Emergency Unit of the University Hospital of Ribeirão Preto, São Paulo, Brazil. This is a government-funded teaching hospital designated as a center for acute stroke care in the city of Riberão Preto (population of approximately 550,000 people). No inpatient rehabilitation services are available for stroke patients, and the standard of care is twice a week outpatient therapy. Eighty-five consecutively admitted stoke patients were screened for eligibility to accrue 20 study participants.

\section{Study measures}

Fugl-Meyer $^{14}$

In the UE subscale of the Fugl-Meyer (FM), the patient is asked to make movements that are considered to reflect the sequential stages of flexion and extension synergies, and the ability to perform selective movements. The section consists of 32 items, which represent movement components, rated on a three-point ordinal scale, $0-2$. The maximum score is 66 . Reliability and validity are well documented. ${ }^{15}$

\section{Action Research Arm Test ${ }^{16}$}

The Action Research Arm Test (ARA) is a scale for functional assessment of strength and coordination. The ARA includes 19 items divided into four subscales: grasp, grip, pinch, and gross movement. Reliability, construct validity, and predictive validity of the ARA have been well established. ${ }^{17}$ The ARA uses ordinal scoring for each subtest item. Item scores are added together to create subtest and a fullscale score with a maximum score of 57 .

National Institutes of Health Stroke Scale ${ }^{18}$

The National Institutes of Health Stroke Scale (NIHSS) evaluates cognitive, sensory and motor impairments as indicators of stroke severity. This 13-item test results in scores ranging from 0 (no deficit) to 46 (severe deficit). Its psychometric properties have been established. ${ }^{19}$

\section{Barthel Index ${ }^{20}$}

The Barthel Index (BI) is a widely accepted measure of ADL function used in stroke. The Barthel Index includes the ten most common areas of ADL function. The primary goal of the $\mathrm{BI}$ is to document the level of independence achieved in basic ADL functions such as bathing, dressing grooming, transfers, ambulation, bowel and bladder function, stairs and toilet use. Reliability and validity are well documented. ${ }^{21}$ Scoring occurs on a 0-100 point scale, wherein a higher score indicates a higher level of functional independence.

\section{Study procedures}

\section{Collection of study measurements}

The inpatient rehabilitation group (in the U.S.) was examined within 24 hours of rehabilitation hospital admission and within 24 hours before discharge. The outpatient group (in Brazil) was examined during 2 home visits, and every effort was made to evaluate the participants between days 7 and 12 for the initial time point, and between days 14 and 30 for the second time point. Inpatients were assessed at the hospital bedside, and outpatients were assessed in their homes.

Study measures were applied in a standardized fashion. The NIHSS was performed with the participant lying in bed, either in their hospital bed or in their bedroom at home. The FM and ARA were performed in the seated position with the chair and table heights adjusted for each patient in the most appropriate position to take their meals.

\section{Rehabilitation interventions}

The inpatient rehabilitation group (in the U.S.) stayed in the rehabilitation inpatient unit for an average of 22 days and typically received 3-5 hours per day of therapies Monday through to Friday, 1-2 hours on Saturday, and none on Sundays. Specialist physician rehabilitation rounds were made 5-6 days per week. Rehabilitation included physical, speech and occupational therapy sessions. The interdisciplinary team also included a psychologist, social worker and nutritionist. Participants were evaluated weekly in team meetings.

The outpatient rehabilitation group was discharged to their homes once neurologically stable. According to the local standard of care, they underwent outpatient rehabilitation twice a week for a total of six hours of physical therapy per patient. Therapists used a neuro-developmental approach..$^{22}$ No patient had speech or occupational therapy treatment.

\section{Data analysis}

\section{Cohort selection}

Demographic characteristics were compared in the two groups: age, sex, race, years of education, living alone, affected side, presence of hemi-inattention, global neurological impairment, independence on the ADL scale, UE impairment and disability, days between stroke onset and first evaluation, days between first and second evaluation, previous BI, medical comorbidities and 0xfordshire classification. ${ }^{23}$ The following variables were dichotomized: living alone, hemi-inattention, lacunar infarct, presence of hypertension, diabetes mellitus, ischemic heart disease, atrial fibrillation, congestive heart failure, previous stroke, smoking and alcoholism. No patients in either cohort had rt-PA treatment during the acute phase.

\section{Statistical analysis}

Student's t-test was used to compare the differences in age, education, days between stroke and first evaluation by NIHSS, FM and BI. For the variables "days between the first and the second evaluation" and the ARA test, which were not normally distributed, nonparametric testing was applied (MannWhitney test). The $\chi^{2}$ and Fisher's exact test were applied to compare race, sex, living alone, affected side, presence of hemi-inattention, 0xfordshire classification and co-morbidities. Fisher's test was used when the criteria for $\chi^{2}$ testing were not fulfilled.

Changes in the impairment and disability scales from baseline to the end of treatment, efficiency and effectiveness were calculated. Efficiency was defined as the amount of improvement obtained per day during the time of rehabilitation [(second evaluation-first evaluation) $\div$ number of days between two evaluations]. Effectiveness was defined as the proportion of the improvement obtained during rehabilitation in relation to the maximum potential of recovery [(second evaluation-first evaluation $) \div$ (maximum scale score-first evaluation)x100]..$^{24,25}$

To establish the outcome of each intervention, changes were compared separately for each group to determine if there was a difference between the initial and final time points. Thereafter, changes between groups were compared by analysis of variance for repeated measures (ANOVA). The efficiency and effectiveness of treatment of each population were compared using the t-test for independent measures. Results were considered to be significant if $p<0.05$. All data analyses were computed using the software SPSS for Windows.

\section{Results}

The baseline characteristics of the two 
groups are shown in Table 1. There was no significant difference in age, sex, race, living alone or previous BI between the two groups $(p>0.05)$. A 10-year difference in education (in years of education) was observed $(p<0.001)$. There were no significant differences in the intervals from stroke onset to first evaluation, or from first to second study evaluation. The incidence of co-morbidities was similar for both groups $(p>0.05)$.

Table 2 shows that stroke characteristics were well matched at the time of the first evaluation. There were no significant differences in stroke severity (measured by the total NIHSS), or stroke type (Oxfordshire Stroke Classification). Both groups had moderate impairment, as shown by a mean NIHSS of $6.7 \pm 3.8$ for the inpatient group and a mean of $8.6 \pm 4.7$ for the outpatient group $(p=0.17)$. There were no significant differences between the two groups in UE motor impairment (FMUE), UE functional limitation (ARA), or basic activities of daily living (BI).

Table 3 reveals that when analyzed separately, the inpatient and outpatient groups had significant improvement from the first to the second evaluation as detected by the section for UE of the FM and ARA test, NIHSS and BI $(p<0.001)$. In addition, the outcome changes for efficiency and effectiveness (section for UE in FM, ARA test, NIHSS and BI) between treatments did not reveal any significant difference between the two groups $(p>0.05)$ (Table 4).

\section{Discussion}

This was a "proof of concept" study, designed to determine whether a study comparing two different rehabilitation strategies in two different countries is feasible. We have demonstrated that a cohort comparison study design can yield two populations treated under substantially different circumstances, which nonetheless resemble each other on most important demographic and clinical variables thought to determine stroke outcome.

The results of this pilot study do not support the hypothesis that moderately affected stroke patients benefit from intensive multidisciplinary inpatient rehabilitation as opposed to outpatient physical therapy. Our results also agree with studies and meta-analyses that concluded that early discharge from hospital with home rehabilitation (early supported discharge) can be as effective as inpatient rehabilitation. ${ }^{8-10,12}$ The main difference is that the present study compared inpatient rehabilitation with nonorganized outpatient rehabilitation twice a week, and the others compared inpatient rehabilitation with organized daily outpatient rehabilitation.

One of the main goals of rehabilitation is to

Table 1. Demographic characteristics of the inpatient and outpatient groups.

\begin{tabular}{lccc} 
& Inpatient $(\mathrm{N}=20)$ & Outpatient $(\mathrm{N}=20)$ & $p$ \\
Age (mean yr, \pm SD) & $68.2 \pm 13.0$ & $64.0 \pm 13.0$ & $0.31^{*}$ \\
Sex (\% male) & 65 & 65 & $1.00^{\text {s }}$ \\
\hline Race (\%) & & & \\
$\quad$ Caucasian & 65 & 85 & $0.14^{\S}$ \\
$\quad$ Black & 35 & 15 & $<0.001^{*}$ \\
Education (mean yr, \pm SD) & $12.4 \pm 3.6$ & $2.7 \pm 2.7$ & $0.74^{\S}$ \\
\hline Living alone (\%) & 35 & 30 & $0.10^{\S}$ \\
Pre-stroke BI $\geq 95$ (\%) & 80 & 100 & $0.12^{*}$ \\
\hline Interval, stroke onset to first & $9.2 \pm 3.7$ & $7.4 \pm 3.5$ & \\
evaluation (mean d., \pm SD) & & & $0.39^{*}$ \\
Interval, first and second & $22.0 \pm 10.4$ & $22.5 \pm 9.2$ & \\
evaluation (mean d., \pm SD) & & & $0.16^{\S}$ \\
Medical co-morbidities (\%) & & & $0.69^{*}$ \\
$\quad$ Hypertension & 60 & 80 & $0.34^{*}$ \\
Diabetes & 25 & 15 & $0.66^{*}$ \\
Coronary disease & 20 & 5 & $1.00^{*}$ \\
Atrial fibrillation & 10 & 20 & $0.31^{\S}$ \\
Heart failure & 10 & 15 & $0.74^{*}$ \\
Previous stroke & 40 & 25 & $1.00^{*}$ \\
Smoking & 35 & 30 & \\
Chronic alcohol abuse & 5 & 5 & \\
\hline
\end{tabular}

't-test for independent samples; ${ }^{+}$Mann Whitney test; *Fischer's exact test; ${ }^{\circledR} \mathrm{X}^{2}$ test; BI: Barthel Index

Table 2. Clinical/neurological status at the time of study enrollment.

\begin{tabular}{lccc} 
& Inpatient $(\mathrm{N}=20)$ & Outpatient $(\mathrm{N}=20)$ & $p$ \\
FM-UE (mean, \pm SD) & $33.4 \pm 22.7$ & $28.0 \pm 25.5$ & $0.48^{*}$ \\
ARA (mean, \pm SD) & $26.3 \pm 23.1$ & $20.8 \pm 22.9$ & $0.40^{*}$ \\
\hline NIHSS (mean, \pm SD) & $6.7 \pm 3.8$ & $8.6 \pm 4.7$ & $0.17^{*}$ \\
Barthel Index (mean, \pm SD) & $55.7 \pm 20.6$ & $49.0 \pm 29.8$ & $0.40^{*}$ \\
\hline Side affected (\% right) & 35 & 30 & $0.73^{\S}$ \\
Neglect (\%) & 30 & 30 & $1.00^{\S}$ \\
\hline Oxford classification(\%) & & & \\
Lacunar & 50 & 35 & $0.33^{*}$ \\
Partial anterior & 30 & 40 & $0.50^{*}$ \\
Total anterior & 15 & 20 & $1.00^{*}$ \\
Posterior & 5 & 5 & $1.00^{*}$ \\
\hline
\end{tabular}

it -test for independent samples; ${ }^{\mathrm{M}}$ Mann Whitney test; *Fischer exact test; $\mathrm{X}^{2}$ test

FM-UE, Fugl-Meyer upper extremity subscale; ARA, action research arm test; NIHSS, National Institutes of Health Stroke Scale.

Table 3. Comparison of pre- and post-treatment outcome scores measure.

\begin{tabular}{lcccccc} 
& \multicolumn{3}{c}{ Inpatient $(\mathrm{N}=20)$} & & \multicolumn{3}{c}{ Outpatient $(\mathrm{N}=20)$} & \\
& Pre-treatment & Post-treatment & $\mathrm{p}$ & Pre-treatment & Post-treatment & p \\
FM-UE & $33.4 \pm 22.7$ & $40.0 \pm 21.9$ & $<0.001$ & $28.0 \pm 25.5$ & $36.6 \pm 23.8$ & $<0.001$ \\
ARA & $26.3 \pm 23.1$ & $32.3 \pm 25.0$ & $<0.001$ & $20.8 \pm 22.9$ & $27.0 \pm 23.6$ & $<0.001$ \\
\hline NIHSS & $6.7 \pm 3.8$ & $4.5 \pm 3.2$ & $<0.001$ & $8.6 \pm 4.7$ & $5.8 \pm 5.4$ & $<0.001$ \\
BI & $55.7 \pm 20.6$ & $73.7 \pm 20.9$ & $<0.001$ & $49.0 \pm 29.8$ & $66.5 \pm 29.5$ & $<0.001$ \\
\hline
\end{tabular}

FM-UE: Fugl-Meyer upper extremity subscale; ARA: action research arm test; NIHSS: National Institutes of Health Stroke Scale; BI: Barthel Index.

promote the reinsertion of the patient in the community. Based on this goal, it has been proposed that home rehabilitation has a better chance to reach these objectives. ${ }^{8}$ At home, patients are forced to face real challenges in daily life while hospital stay can determine physical immobility, impairment of family relationships and social isolation. ${ }^{26}$
Our results must be interpreted with caution. Substantial effort was made to collect and match representative samples from both locations, but the small number of subjects in each sample might not have been sufficient to reveal a significant difference between the two groups. Therefore, type II error, due to limited sampling is an alternative explanation for the 
Table 4. Changes of the outcome measures among the inpatient (U.S.) and outpatient (Brazil) rehabilitation treatments.

\begin{tabular}{lccc} 
& Inpatient $(\mathrm{N}=20)$ & Outpatient $(\mathrm{N}=20)$ & $p$ \\
Change & & & \\
FM-UE & $6.6 \pm 7.9$ & $8.6 \pm 9.0$ & $0.36^{\infty}$ \\
ARA & $5.9 \pm 8.9$ & $6.2 \pm 8.0$ & $0.91^{\infty}$ \\
NIHSS & $2.1 \pm 1.6$ & $2.8 \pm 1.8$ & $0.26^{\infty}$ \\
BI & $18.0 \pm 11.2$ & $17.5 \pm 16.9$ & $0.91^{\infty}$ \\
Efficiency (mean, \pm SD) & & \\
FM & $0.23 \pm 0.11$ & $0.29 \pm 0.10$ & $0.81^{*}$ \\
ARA & $0.19 \pm 0.06$ & $0.21 \pm 0.01$ & $0.96^{*}$ \\
NIHSS & $0.10 \pm 0.08$ & $0.13 \pm 0.08$ & $0.21^{+}$ \\
BI & $0.91 \pm 0.63$ & $0.84 \pm 0.83$ & $0.76^{+}$ \\
\hline Effectiveness (\%) & & & \\
FM & $26.4 \pm 20.8$ & $33.3 \pm 29.1$ & $0.39^{+}$ \\
ARA & $18.1 \pm 3.51$ & $23.1 \pm 0.44$ & $0.60^{*}$ \\
NIHSS & $33.0 \pm 24.1$ & $44.7 \pm 33.3$ & $0.20^{+}$ \\
BI & $51.2 \pm 35.6$ & $42.4 \pm 37.2$ & $0.46^{+}$ \\
\hline
\end{tabular}

${ }^{\infty}$ Analysis of variance for repeated measures; +t-test for independent samples; *Mann Whitney test; FM-UE, Fugl-Meyer upper extremity subscale; ARA, action research arm test; NIHSS, National Institutes of Health Stroke Scale; BI, Barthel Index;

lack of statistical difference between the two groups studied. Another factor to be considered is that most of the study participants had mild impairment and these patients have a good prognosis regardless of the intervention. ${ }^{27}$ Also, one month of follow-up may not have been sufficient to observe a long-term beneficial effect.

An important methodological concern in selecting subjects from different countries is appropriate matching for key clinical characteristics such as the NIHSS, BI, FM, ARA, Barthel index, Oxfordshire classification and hemi-inattention. Despite the lack of a significant difference between the two groups, the outpatient group could have been favored by the inclusion of patients with more severe stroke, therefore with more potential for recovery. The American group had more stroke subtypes following the 0xfordshire Classification with involvement of the anterior circulation than the Brazilian group. Additionally, the prestroke BI score was less than 100 in four U.S. patients, but not in any in the Brazilian cohort. The Brazilian group might also have been favored in the first evaluation since it was examined on average two days before the inpatient group. This fact might have caused a favorable effect for the outpatient group since recovery is faster during the first days after stroke. $^{28,29}$

Our preliminary results also highlight the need for studies comparing different rehabilitation strategies, since the superiority of inpatient rehabilitation in this stroke population could not be confirmed. Recruiting participants from countries with different standards of care allows investigators to explore the effectiveness of specific rehabilitation regimens and reduce concerns about withholding usual and customary care.

\section{References}

1. Rosamond W, Flega K, Furie K, et al. Heart disease and stroke statistics-2008 update: a report from the American Heart Association Statistics Committee and Stroke Statistics Subcommittee. Circulation 2008;117:e-24-146.

2. Smith JP. Healthy bodies and thick wallets: the dual relation between health and economic status. J Econ Perspect 1999;13:14466.

3. Starfield B. Equity and health: a perspective on nonrandom distribution of health in the population. Rev Panam Salud Publica 2002;12:384-7.

4. Ruger JP, Kim HJ. Global health inequalities: an international comparison. J Epidemiol Community Health 2006;60:928-36.

5. Fiedler RC, Granger CV, Russel CF. UDS(MR)SM: follow-up data on patients discharged in 1994-1996. Uniform Data System for Medical Rehabilitation. Am J Phys Med Rehabil 2000;79:184-92.

6. Langhorne P, Pamela D. Does the organization of postacute stroke care really matter? Stroke 2001;32:268-74.

7. Stroke Unit Trialists' Collaboration. Collaborative systematic review of the randomised trials of organised inpatient (stroke unit) care after stroke. Br Med J 1997;314:1151-9.

8. Langhorne P, Taylor G, Murray GD, et al. Early supported discharge services for stroke patients: a meta-analysis of individual patient's data. Lancet 2005;365:501-6.

9. Legg L, Langhorne P, Outpatients Services Trialists. Rehabilitation therapy services for stroke patients living at home: systematic review of randomized trials. Lancet
2004;363:352-6.

10. Holmqvist LW, von Koch L, Pedro-Cuesta J. Use of healthcare, impact on family caregivers and patient satisfaction of rehabilitation at home after stroke in southwest Stockholm. Scand J Rehabil Med 2000;32: 173-9.

11. Anderson C, Mhurchu CN, Brown PM, Carter K. Stroke rehabilitation services to accelerate hospital discharge and provide home-based care: an overview and cost analysis. Phamacoeconomics 2002;20:53752.

12. Fjaertoft H, Indredavick B, Magnussen $\mathrm{J}$, Johnsen R. Early supported discharge for stroke patients improves clinical outcome. Does it also reduces use of healthy services and costs? One-year follow-up of a randomized controlled trial. Cerebrovasc Dis 2005;19:376-83.

13. Langhorne P, Holmqvist LW, Early Supported Discharge Trialists. Early supported discharge after stroke. J Reabil Med 2007;39: 103-8.

14. Fulg-Meyer A, Jaasko L, Leyman I, et al. The post-stroke hemiplegic patient: A method of evaluation of physical performance. Scand J Rehab Med 1975;7:13-31.

15. Duncan P, Propst M, Nelson S. Reliability of the Fulg-Meyer Assessment of sensorimotor recovery following cerebrovascular accident. Phys Ther 1983;63:1606-10.

16. Lyle RC. A performance test for assessment of upper limb function in physical rehabilitation treatment and research. Intern J Rehabil Res 1981;4:483-92.

17. De Weerdt WJC, Harrison MA. Measuring recovery of arm-hand function in stroke patients: A comparison of the BrunnstromFugl-Meyer test and the Action Research Arm test. Physiother Canada 1985;37:65-70.

18. Brott T, Adams HP, Olinger CP, et al. Measurements of acute cerebral infarction: a clinical examination scale. Stroke 1989;20:864-70.

19. Muir KW, Weir CJ, Murray GD, et al. Comparison of neurological scales and scoring systems for acute stroke prognosis. Stroke 1996;27:1817-20.

20. Mahoney FI, Barthel DW. Functional Evaluation: the Barthel Index. Md State Med Journal 1955;14:61-5.

21. Collin C, Wade DT, Davies S, Horne V. The Barthel ADL Index: a reliability study. Int Disabil Stud 1988;10:61-3.

22. Bobath B. Adult hemiplegia. Evaluation and treatment. Oxford: Heinemann Medical, 1990.

23. Bamford J, Sandercock P, Dennis M, et al. Classification and natural history of clinically identifiable subtypes of cerebral infarction. Lancet 1991;337:1521-6.

24. Heinemann AW, Roth EJ, Cichowski K, Betts HB. Multivariate analysis of 
improvement and outcome following stroke rehabilitation. Arch Neurol 1987;44: 1167-72.

25. Shah S, Vanclay F, Cooper B. Efficiency, effectiveness, and duration of stroke rehabilitation. Stroke 1990;21:241-6.

26. Mayo NE, Wood-Dauphinee S, Côté R, et al. There's no place like home. An evalua- tion of early supported discharge for stroke. Stroke 2000;31:1016-23.

27. Adams HP, Davis PH, Leira EC, et al. Baseline NIH stroke scale score strongly predicts outcome after stroke. A report of the Trial of Org 10172 in Acute Stroke Treatment (TOAST). Neurology 1999;53: 126-31.
28. Jorgensen HS, Nakayama H, Raaschou HO, et al. Outcome and time course of recovery in stroke. Part I: Outcome. The Copenhagen Stroke Study. Arch Phys Med Rehabil 1995;76:399-405.

29. Wade DT, Wood VA, Hewer RL. Recovery after stroke - The first 3 months. J Neurol Neurosurg Psychiatry 1985;48:7-13. 